\title{
Dual Self-Paced Graph Convolutional Network: Towards Reducing Attribute Distortions Induced by Topology
}

\author{
Liang Yang ${ }^{1,2}$, Zhiyang Chen ${ }^{1,2}$, Junhua Gu ${ }^{1,2, *}$ and Yuanfang Guo ${ }^{3}$ \\ ${ }^{1}$ School of Artificial Intelligence, Hebei University of Technology, China \\ ${ }^{2}$ Hebei Province Key Laboratory of Big Data Calculation, Hebei University of Technology, China \\ ${ }^{3}$ School of Computer Science and Engineering, Beihang University, China \\ yangliang@nextseason.cc, valarzychen@gmail.com,jhgu@hebut.edu.cn, andyguo@buaa.edu.cn
}

\begin{abstract}
The success of graph convolutional neural networks (GCNNs) based semi-supervised node classification is credited to the attribute smoothing (propagating) over the topology. However, the attributes may be interfered by the utilization of the topology information. This distortion will induce a certain amount of misclassifications of the nodes, which can be correctly predicted with only the attributes. By analyzing the impact of the edges in attribute propagations, the simple edges, which connect two nodes with similar attributes, should be given priority during the training process compared to the complex ones according to curriculum learning. To reduce the distortions induced by the topology while exploit more potentials of the attribute information, Dual Self-Paced Graph Convolutional Network (DSP-GCN) is proposed in this paper. Specifically, the unlabelled nodes with confidently predicted labels are gradually added into the training set in the node-level self-paced learning, while edges are gradually, from the simple edges to the complex ones, added into the graph during the training process in the edge-level self-paced learning. These two learning strategies are designed to mutually reinforce each other by coupling the selections of the edges and unlabelled nodes. Experimental results of transductive semi-supervised node classification on many real networks indicate that the proposed DSP-GCN has successfully reduced the attribute distortions induced by the topology while it gives superior performances with only one graph convolutional layer.
\end{abstract}

\section{Introduction}

Graph partitioning has contributed tremendously in both the traditional unsupervised node classification techniques and the latest semi-supervised node classification approaches [Kipf and Welling, 2017; Veličković et al., 2018]. From either the spectral or spatial perspectives, many graph convolutional neural networks (GCNNs) have been proposed recently

\footnotetext{
${ }^{*}$ Corresponding author.
}

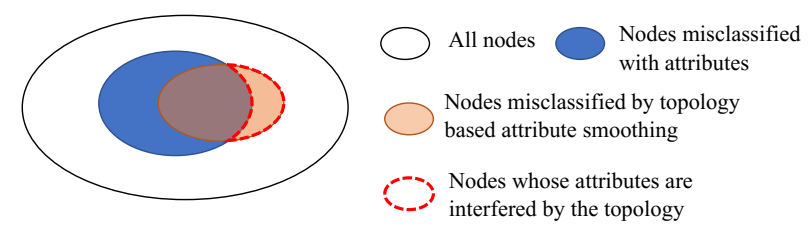

Figure 1: Different types of misclassified nodes. The nodes may be misclassified with respect to the original attributes only. It may also be misclassified by topology based attribute smoothing. The topology will correct certain incorrect classifications, while interfere some correct predictions. This distortion will induce a certain amount of misclassifications (the area surrounded by the red dashed lines) of the nodes.

[Defferrard et al., 2016; Hamilton et al., 2017]. By incorporating supervised information, GCNN based semi-supervised node classification methods have significantly improved the performances of the traditional unsupervised techniques.

Similar to other semi-supervised learning tasks [Chapelle et al., 2009; Zhu, 2006], semi-supervised node classification requires efficient incorporations of the unsupervised information. When classifying the nodes in the attributed graphs, the attributes of the unlabelled nodes serve as the unsupervised information. Due to the sparsity of the node attributes, prediction only based on the original node attributes cannot fully exploit their relationships and usually results in overfittings. Therefore, many GCNNs [Kipf and Welling, 2017; Veličković et al., 2018] perform operations which are equivalent to the attribute smoothing (propagating) over the graph [Li et al., 2018]. Therefore, GCNNs actually utilizes the unsupervised information by augmenting the node attributes in local neighbourhoods.

Unforunately, this propagation strategy possesses certain weaknesses. Typically, over propagation may degrade the prediction performance [Li et al., 2018]. Since the over propagation tends to amend all the nodes to have the identical augmented attributes which certainly reduces the discriminabilities of the nodes. Therefore, existing GCNNs only employ two stacked graph convolutional layers, i.e., two-hop propagations to prevent the over propagation. Besides, the attributes may be interfered by the utilization of the topology information. This distortion will induce a certain amount of misclassification of the nodes, which can be correctly predicted with only the attributes, as illustrated in Fig. 1. 
In this paper, we intend to reduce the negative effects, i.e., the distortions, caused by the utilization of the topology. Thus, the impact of the edges in attribute propagations is analyzed. As elaborated in Fig. 2, there exists two types of edges. If the two nodes $v_{i}$ and $v_{j}$, whose attributes are similar, are connected by an edge (green line), the attributes of each node will vary slightly after propagations. Since this kind of edges usually contain less information and the network can quickly learn from them, we denote them as the simple edges. If the two nodes $v_{i}$ and $v_{k}$, whose attributes are quite different, are connected by an edge (red line), the attributes of each node will be significantly changed after propagations. This kind of edges contain rich information and the network usually requires more trainings to learn from them. Therefore, these edges are denoted as the complex edges.

Spontaneously, a question can be raised that whether these two kinds of edges should be learned simultaneously by the network in the training stage? Curriculum Learning (CL [Bengio et al., 2009]) and Self-Paced Learning (SPL [Kumar et al., 2010]), which mimic the learning process of human beings, conclude that by learning from simple concepts to complex ones, the learning of the network can be significantly improved. Based on this philosophy, we believe that the topology information should be evaluated and feed into the network gradually from the simple edges to the complex ones during the training process of GCNNs.

To reduce the distortions generated by utilizing the topology information while exploit more potentials of the attribute information, Dual Self-Paced Graph Convolutional Network (DSP-GCN) is proposed in this paper. Specifically, the unlabelled nodes with confidently predicted labels are gradually included into the training set in the node-level self-paced learning to better exploit the attribute information. To reduce the distortions when utilizing the topology information, edges are gradually, from the simple edges to the complex ones, added into the graph during the training process in the edge-level self-paced learning. Since the nodes and edges are highly correlated, the node-level and edge-level self-paced learnings are also correlated. Therefore, these two learning strategies are designed to mutually reinforce each other by coupling the selections of the edges and unlabelled nodes. If an unlabelled node is included during the training process, its edges will likely to be included. On the contrary, if most of the edges of an unlabelled node is added, this node as well as its predicted label tend to be put into the training with a high probability.

Our contributions are summarized as below:

- We observe that the utilization of the topology in GCNNs tends to interfere the correct prediction results which can be obtained with respect to the attributes only, according to the experiments.

- We propose an edge-level self-paced learning strategy by gradually train the network from the simple edges to the complex ones to reduce the negative effects caused by the utilization of the topology, instead of feeding the entire topology directly into the network.

- We propose a Dual Self-Paced Graph Convolutional Network (DSP-GCN) which jointly exploits the node-

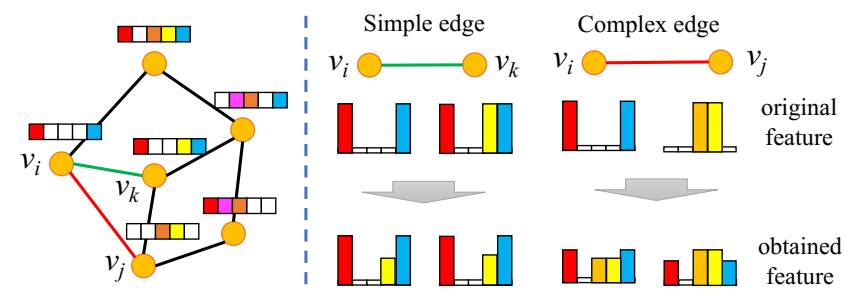

Figure 2: Simple and complex edges. A simple edge (green line) connects two nodes which contain similar attributes. After smoothing over the simple edge, their node attributes do not vary significantly. A complex edge (red line) connects two nodes which contain much different attributes. After smoothing over the complex edge, the discriminabilities of the node attributes are reduced.

level and edge-level self-paced learning strategies.

- Experimental results on eight real networks indicate that DSP-GCN can successfully reduce the distortion induced by the topology and yield superiority performances with only one graph convolutional layer.

\section{Preliminaries}

\subsection{Notations}

A network can be represented by an attributed graph $G=$ $(V, E, X) . \quad V=\left\{v_{i} \mid i=1, \ldots, N\right\}$ is a set of $|V|=N$ vertices, where $v_{i}$ is associated with a feature $x_{i} \in \mathbb{R}^{K}$. $X \in \mathbb{R}^{N \times K}$ represents the collection of the features. Each row of $X$ corresponds to a node. $E$ stands for a set of edges. Each of the edges connects two vertices in $V$. The adjacency matrix $A=\left[a_{i j}\right] \in \mathbb{R}^{N \times N}$ is obtained according to the network topology. If an edge connects the vertices $v_{i}$ and $v_{j}$, $a_{i j}=1$, and vice versa. If self-edges are allowed in the network, then $a_{n n}=1$. Otherwise $a_{n n}=0 . a_{n}$, which denotes the $n^{\text {th }}$ column of $A$, can be utilized to represent the local neighbourhood of the vertex $v_{n} . d_{n}=\sum_{j} a_{n j}$ stands for the degree of $v_{n}$ while $D=\operatorname{diag}\left(d_{1}, d_{2}, \ldots, d_{N}\right)$ is the degree matrix of $A$. The graph Laplacian and its normalized form are defined as $L=D-A$ and $L=D^{-\frac{1}{2}} L D^{-\frac{1}{2}}$, respectively. For a network, given the labels $Y \in \mathbb{R}^{\left|V_{l}\right| \times F}$ (where $F$ represents the number of classes), which belong to a set of vertices $V_{l} \subset V$, a typical semi-supervised node classification algorithm classifies other nodes in $V-V_{l}$ according to the attributed graph. For simplicity, the first $l$ nodes $\left\{v_{i}\right\}_{i=1}^{l}$ are assumed to be labelled.

\subsection{Graph Convolutional Network}

By simplifying the complex existing models, Graph Convolutional Network (GCN) [Kipf and Welling, 2017] defines the graph convolution operation as

$$
H_{G C N}=\tilde{D}^{-\frac{1}{2}} \tilde{A} \tilde{D}^{-\frac{1}{2}} X
$$

where $\tilde{A}=A+I_{N}$ and $\tilde{D}_{n n}=\sum_{j} \tilde{A}_{n j}=d_{n}+1$. Then, the to-be-predicted labels can obtained by feeding $H_{G C N}$ into a fully-connected layer as

$$
T_{G C N}=H_{G C N} W .
$$


By minimizing the cross-entropy, which is defined in Eq. (3), between the predictions and given labels, the parameter $\mathrm{W}$ can be obtained.

$$
\mathcal{L}=-\sum_{n \in V_{l}} \sum_{f=1}^{F} y_{n f} \log \left(t_{n f}\right)
$$

[Li et al., 2018] concludes the mechanism and success of GCN that it is equivalent to perform a symmetric Laplacian smoothing $\left(H_{G C N}=\tilde{D}^{-\frac{1}{2}} \tilde{A} \tilde{D}^{-\frac{1}{2}}\right)$ operation before the actual predictions. It aims at augmenting the attributes with topology to alleviate the problem of attribute sparsity. The performance of the smoothing based classification obviously outperforms the classification based on the original attributes. Unfortunately, the employment of the topology information also induces certain negative effects to the classification results. As illustrated in Fig. 1, some nodes (i.e. the non-overlapped orange set), which can be correctly classified according to the original attributes, are actually misclassified with respect to the smoothed attributes after the attribute propagations.

\subsection{Self-paced Learning}

Inspired by the learning process of human beings, i.e., learning from easy concepts to complex ones, [Bengio et al., 2009] proposes a curriculum learning (CL) strategy which gradually includes the training samples from the easy samples to the complex ones during the training process. Self-paced Learning (SPL [Kumar et al., 2010]) adopts this strategy by incorporating the curriculum design as a regularization term into the learning objective function as

$$
\sum_{i=1}^{N} q_{i} \ell\left(y_{i}, f\left(x_{i}, w\right)\right)-\lambda \sum_{i=1}^{N} q_{i}
$$

where $f\left(x_{i}, w\right)$ represents the prediction function with learnable model parameter $w, \ell\left(y_{i}, f\left(x_{i}, w\right)\right)$ stands for the loss function between the prediction $f\left(x_{i}, w\right)$ and ground truth $y_{i}$, and $q_{i} \in\{0,1\}$ is the sample weight to indicate whether the data point $x_{i}$ has been included in the training process. Note that the second term $\lambda \sum_{i=1}^{N} q_{i}$ is the self-paced regularization term, with $\lambda$ being the pace parameter which controls the learning pace. The model is trained by jointly minimizing the objective function in Eq. (4) with respect to the model parameter $w$ and the sample weights $q_{i}$ 's by gradually increasing the pace parameter $\lambda$. For a fixed $w$, the optimal $q_{i}^{*}$ is

$$
q_{i}^{*}=\left\{\begin{array}{ll}
1 & \text { if } \ell_{i}<\lambda \\
0 & \text { otherwise }
\end{array},\right.
$$

where $\ell_{i}=\ell\left(y_{i}, f\left(x_{i}, w\right)\right)$ represents the loss of predicting the data sample $x_{i}$ as $f\left(x_{i}, w\right)$. With a small $\lambda$, only the data samples with small losses (i.e. easy to be predicted) are included in the training data. When $\lambda$ gradually increases, more complex data samples are added.

Self-paced learning has already been extended to semisupervised learning and unsupervised matrix factorization. Self-paced semi-supervised learning augments the training set with the unlabelled data samples based on the confidence predictions [Ma et al., 2017]. The unlabelled data samples are gradually included by combining the self-paced strategy and co-training of two views. Meanwhile, self-paced matrix factorization extends the previous self-paced learning strategy which is designed for the data samples to a self-paced learning for the pairs of data samples and their corresponding attributes [Zhao et al., 2015].

\section{Self-paced Network Embedding}

Recently, self-paced learning is applied to network embedding. [Zhou et al., 2018] follows the co-training strategy in [Ma et al., 2017] and extends the co-training of two views to two tasks, i.e., embedding and prediction. However, this node-level self-paced strategy cannot reduce the negative impact to the attributes induced by the topology. [Gao and Huang, 2018] adopts the self-paced strategy to select the negative samples and performs a self-paced training with the pair of data samples similar to [Zhao et al., 2015]. Unfortunately, self-paced negative sampling requires to compute the similarities between almost all the pairs of nodes, which is computationally inefficient. Besides, similar to other semi-supervised node classification methods introduced above, these two methods also ignore the impact of topology in the self-paced node selections.

\section{Proposed Work}

In this section, Dual Self-Paced Graph Convolutional Network (DSP-GCN), which reduces the topology distortions on the attributes, is proposed. DSP-GCN consists of two interactive self-paced learning strategies, node-level and edge-level strategies. The node-level self-paced strategy gradually includes the unlabelled nodes, whose labels are predicted with high confidence scores, into the training set. The edge-level self-paced strategy gradually incorporates the edges, from the simple edges to the complex ones, into the graph convolutional operation of GCN. Note that both the strategies will mutually reinforce each other, because the nodes and edges in the graph is usually highly correlated. If an unlabelled node is added into the training set, its edges tend to be included. Meanwhile, if most of the edges of an unlabelled node has already been included, this node as well as its predicted label will likely to be added into training set.

\subsection{Node-level Self-Paced Learning}

Our node-level self-paced learning augments the training set to fully exploit the unsupervised information (i.e., the attributes of the unlabelled nodes). Since we gradually add the unlabelled nodes, which possess the predicted labels with high confidence scores, into the training set, the objective function can be formulated as

$$
\begin{aligned}
\mathcal{L}_{\text {node }}=\sum_{i=1}^{l} \ell\left(y_{i}, g\left(x_{i}, A, W\right)\right) & +\sum_{k=l+1}^{N} q_{k} \ell\left(y_{k}, g\left(x_{k}, A, W\right)\right) \\
& -\lambda_{\text {node }} \sum_{k=l+1}^{N} q_{k}
\end{aligned}
$$

where the three terms at the right-hand side of the equation are a supervised loss, an unsupervised loss and a self-paced 
regularizer, respectively. $y_{i}$ and $y_{k}$ denote the given labels of the labelled nodes and the predicted labels of the unlabelled nodes, respectively. $q_{k} \in\{0,1\}$ is the weight assigned to the unlabelled node $v_{k}$ to indicate whether it as well as its predicted label $y_{k}$ has been added into the training set. $\lambda_{\text {node }}$ is the pace parameter which controls the learning pace. If $\lambda_{\text {node }}$ increases, more unsupervised nodes will be added into the training set, i.e., more unsupervised nodes satisfy the condition of $q_{k}^{*}=1$, and vice versa. The prediction model $g\left(x_{k}, A, W\right)$ is identical to GCN as shown in Eqs. (1) and (2), and the loss function $\ell(.,$.$) is the cross-entropy as shown$ in Eq. (3). With a fixed $W$, minimizing Eq. (6) with respect to $q_{k}$ gives the same optimal solution as Eq. (5) presents.

\subsection{Edge-level Self-Paced Learning}

Unfortunately, optimizing Eq. (6) with a fixed topology $A$ is equivalent to directly add all the edges (i.e., the entire topology) into the training of GCN, which may induce certain negative effects to the attributes as elaborated in Fig. 1. To alleviate this issue, the negative impact of the topology to the discriminabilities of the node attributes is considered and formulated. Since there exists different kinds of edges, which possess different degrees of training difficulties and have been illustrated in Sec. 1 and Fig. 2, we propose to exploit the self-paced learning strategy [Kumar et al., 2010] to gradually include edges with different degrees of training difficulties (from the simple edges to the complex ones) into the training process. To measure the difficulty of learning from the edge $e_{i j}$, we exploit the attribute variations on the nodes $v_{i}$ and $v_{j}$ after the attribute propagations as the measurement, which also indicates the amount of information possessed by $e_{i j}$. This quantity can be described as the inner product of the attributes of nodes $v_{i}$ and $v_{j}$ as

$$
s_{i j}=1-\frac{\exp \left(x_{i} B x_{j}^{T}\right)}{\sum_{k \in N(i)} \exp \left(x_{i} B x_{k}^{T}\right)},
$$

where $B$ is the learnable parameter to measure the consistency between the corresponding attributes of the two nodes. For example, "learning" and "inference" are two similar attributes in machine learning community. If the attribute "learning" is propagated to a node $v_{i}$ which possesses the attribute "inference" yet without the attribute 'learning", the attribute amendment occurred on node $v_{i}$ tends to be minor and only slightly increases the entropy of node $v_{i}$.

Instead of adding a self-paced regularizer as the node-level strategy, our edge-level self-paced learning strategy directly selects the edges which are included in the training process via

$$
\hat{a}_{i j}= \begin{cases}1 & \text { if } s_{i j}<\lambda_{e d g e} \\ 0 & \text { otherwise }\end{cases}
$$

where $\hat{a}_{i j}$ is edge weight and $\lambda_{\text {edge }}$ is the pace parameter to control the learning pace of the edges. When $\lambda_{e d g e}$ is small, only the edges $e_{i j}$ with high $s_{i j}$, which will not significantly increase the attribute entropy of the connected nodes, is added. When $\lambda_{e d g e}$ increases, more edges will be included.

\subsection{Dual Self-Paced Learning}

The node-level self-paced learning in Eq. (6) and edgelevel self-paced learning in Eq. (8) can be respectively tuned with two pace parameters $\lambda_{\text {node }}$ and $\lambda_{\text {edge }}$. Meanwhile, both strategies will mutually reinforce each other.

Intuitively, if the prediction confidence of an unsupervised node is high, this node will be added into the training set according to the self-paced learning strategy. The high prediction confidence of a node is usually induced by the consistency between it and its neighbourhoods. Besides, this consistency indicates that the edges between them are the simple ones, as shown in Fig. 2. Then, these edges are also added into the graph. On the other hand, the addition of certain edges into the training set may also induce the addition of certain nodes in a similar manner to the above situation.

To model the interactions between the two strategies, the weight $q_{k}$ of unlabelled node and weight $\hat{a}_{i j}$ of edge are jointly considered to form the loss of the interactions as

$$
\mathcal{L}_{\text {inter }}=-\gamma \sum_{k=l+1}^{N}\left(q_{k} \sum_{j \in N(k)} \frac{\hat{a}_{k j}}{\hat{d}_{k}+1}\right),
$$

where $\hat{d}_{k}=\sum_{j} \hat{a}_{k j}$ represents the current degree of the node $v_{k}$ in graph $\hat{A}$. $\hat{d}_{k}+1$, which is equivalent to adding a selfloop to each node as GCN, is employed to avoid the zero divisor. Since the edge-level self-paced learning in Eq. (8) is performed as a constraint instead of a loss term as the nodelevel self-paced learning. A simple combination of the two losses in Eqs. (6) and (9) cannot achieve the reinforcement from the node-level learning to the edge-level learning. To alleviate this issue, Eq. (8) is modified as

$$
\hat{a}_{i j}= \begin{cases}1 & \text { if } s_{i j}<\lambda_{e d g e}+\gamma \frac{q_{i}}{\hat{d}_{i}+1} \\ 0 & \text { otherwise }\end{cases}
$$

If the unlabelled node $v_{i}$ has been added to the training set, i.e., $q_{i}=1$, the probability of edge $e_{i j}$ being included will increase from $\lambda_{\text {edge }}$ to $\lambda_{\text {edge }}+\gamma \frac{q_{i}}{\hat{d}_{i}+1}$.

\subsection{Objective Function and Optimization}

By combining Eqs. (6), (9) and (10), the overall objection function is constructed as

$$
\begin{gathered}
\mathcal{L}=\sum_{i=1}^{l} \ell\left(y_{i}, g\left(x_{i}, \hat{A}, W\right)\right)+\sum_{k=l+1}^{N} q_{k} \ell\left(y_{k}, g\left(x_{k}, \hat{A}, W\right)\right) \\
-\lambda_{\text {node }} \sum_{k=l+1}^{N} q_{k}-\gamma \sum_{k=l+1}^{N}\left(q_{k} \sum_{j \in N(k)} \frac{\hat{a}_{k j}}{\hat{d}_{k}+1}\right) \\
\text { s.t. } \hat{a}_{i j}= \begin{cases}1 & \text { if } s_{i j}<\lambda_{e d g e}+\gamma \frac{q_{i}}{\hat{d}_{i}+1} \\
0 & \text { otherwise }\end{cases}
\end{gathered}
$$

with the hyper-parameters $\gamma, \lambda_{\text {node }}$ and $\lambda_{\text {edge. }}$. The parameters to be learned in Eq. (11) are $W, B, y_{k}, q_{k}$ and $\hat{a}_{i j}$. This objective function can be minimized according to an alternative optimization strategy. The optimization process are 


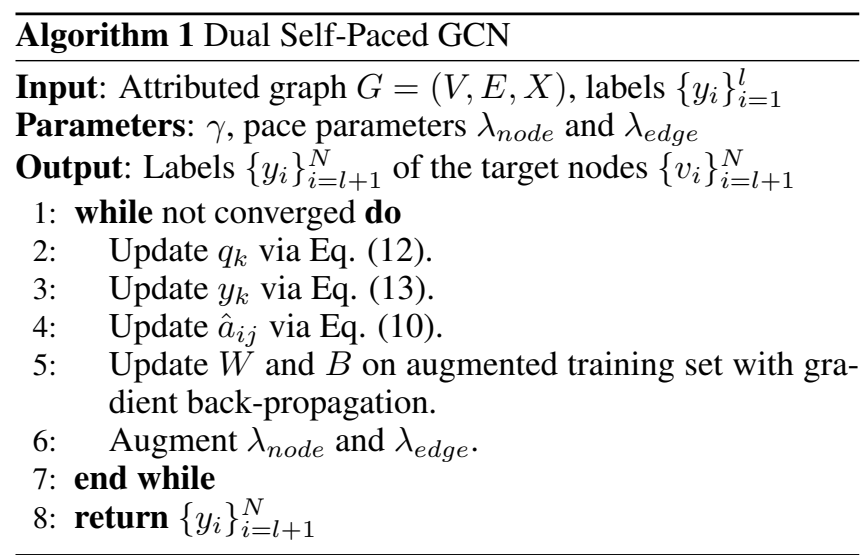

shown in Algorithm 1 with the main steps elaborated as follows:

Update $q_{k}$ : This step is equivalent to minimizing $\mathcal{L}$ in Eq. (11) without the supervised term and the constraint. Since $q_{k} \in\{0,1\}$, its optimal solution is

$$
q_{k}^{*}=\left\{\begin{array}{ll}
1 & \text { if } \ell_{k}<\lambda_{\text {node }}+\gamma \sum_{j}\left(\frac{\hat{a}_{k j}}{\hat{d}_{k}+1}\right) \\
0 & \text { otherwise }
\end{array} .\right.
$$

Here, $\ell_{k}=\ell\left(y_{k}, g\left(x_{k}, \hat{A}, W\right)\right)$ represents the difference between the predictions of two consecutive iterations, where $y_{k}$ stands for the predicted label in the previous iteration, and $g\left(x_{k}, \hat{A}, W\right)$ represents the predicted label in the current iteration according to the new $\hat{a}_{i j}, W$ and $B$. The difference threshold is $\lambda_{\text {node }}+\gamma \sum_{j}\left(\frac{\hat{a}_{k j}}{\hat{d}_{k}+1}\right)$, where the first term $\lambda_{\text {node }}$ is the pace parameter. The second term $\gamma \sum_{j}\left(\frac{\hat{a}_{k j}}{\hat{d}_{k}+1}\right)$ indicates the probability of this node $v_{k}$ being included in the training set, if more edges $\hat{a}_{k j}$ of the unlabelled node $v_{k}$ is included. By updating $q_{k}$, we can improve the self-paced node selections with our self-paced edge selections.

Update $y_{k}$ : Since only the second term of $\mathcal{L}$ in Eq. (11) contains the variable $y_{k}$, the optimal $y_{k}^{*}$ can be obtained by minimizing $\ell\left(y_{k}, g\left(x_{k}, \hat{A}, W\right)\right)$. Therefore,

$$
y_{k}^{*}=g\left(x_{k}, \hat{A}, W\right),
$$

where $g\left(x_{k}, \hat{A}, W\right)$ is identical to GCN as introduced in Eqs. (1) and (2), i.e., label is predicted by GCN with parameters $W, B$ and the topology $\hat{a}_{i j}$ obtained in the previous iteration.

Update $\hat{a}_{i j}$ : The optimal solution of $a_{i j}$ has been shown in Eq. (10). By updating $a_{i j}$, the proposed self-paced edge selections can be further enhanced with respect to the selfpaced node selections.

Update $W$ and $B$ : In this step, we only have to optimize the first two terms of $\mathcal{L}$ in Eq. (11), i.e.,

$$
\sum_{i=1}^{l} \ell\left(y_{i}, g\left(x_{i}, \hat{A}, W\right)\right)+\sum_{k=l+1}^{N} q_{k} \ell\left(y_{k}, g\left(x_{k}, \hat{A}, W\right)\right),
$$

whose parameters are $W$ and $B$. Here, only the unlabelled nodes, which are included in the training set, i.e., $q_{k}=1$, will contribute to the second term. Since the cross-entropy

\begin{tabular}{lrrrr}
\hline Dataset & \#Nodes & \#Edges & \#Classes & \#Features \\
\hline Texas & 187 & 328 & 5 & 1,703 \\
Cornell & 195 & 304 & 5 & 1,703 \\
Washington & 230 & 446 & 5 & 1,703 \\
Wisconsin & 265 & 530 & 5 & 1,703 \\
Wiki & 3,363 & 45,006 & 19 & 4,972 \\
\hline CiteSeer & 3,327 & 4,732 & 6 & 3,703 \\
Cora & 2,708 & 5,429 & 7 & 1,433 \\
PubMed & 19,717 & 44,338 & 3 & 500 \\
NELL & 65,755 & 266,144 & 210 & 5,414 \\
\hline
\end{tabular}

Table 1: Datasets.

in Eq. (3) is adopted as a loss, the gradient back-propagation can be utilized to obtain $W$ and $B$ similar to GCN [Kipf and Welling, 2017] and GAT[Veličković et al., 2018].

Remark 1: Comparing with the existing self-paced network embedding approaches, DSP-GCN possesses two obvious advantages. 1) DSP-GCN considers self-paced learning on edges to reduce the negative effects caused by employing the topology. 2) DSP-GCN considers and models the correlations between the node-level and edge-level self-paced learning to improve the prediction efficiencies.

Remark 2: The graph construction is the updating of $\hat{a}_{i j}$ in Eq. (10) with $s_{i j}$ defined in Eq. (7). The computational complexity of each edge $s_{i j}$ is $O(K)$, where $K$ is the number of features. Therefore, the overall computational complexity of the graph construction is $O(M K)$ where $M$ is the number of edges in the graph, i.e., the computations of the graph construction will linearly increase when the scale of the network increases.

\section{Experimental Results}

In this section, we validate the proposed DSP-GCN by empirically evaluating the performances in the transductive semisupervised node classification task. In DSP-GCN, only one graph convolutional layer is employed instead of the two layers utilized in GCN and GAT. Here, we set $\gamma=1$ and initialize $\lambda_{\text {node }}=0.12$ and $\lambda_{\text {edge }}=0.2$ on all the networks. Note that $\lambda_{\text {node }}$ and $\lambda_{\text {edge }}$ are augmented via two multipliers ranging from 1.05 to 1.11 , respectively.

\subsection{Datasets}

In the experiments, three common citation networks [Sen et al., 2008], Cora, CiteSeer and PubMed, and a knowledge graph NELL [Carlson et al., 2010] are employed as shown in Table 1. In each citation network, papers and undirected citations are defined as the nodes and edges, respectively. The node content is represented by the bag-of-word representation of the documents. Papers are classified into various categories according to their disciplines. A bipartite graph is extracted from the knowledge graph as in [Yang et al., 2016]. Besides of the entity nodes, each relation tuple $\left(e_{i}, r, e_{j}\right)$ is decomposed into two connected relation nodes $\left(e_{i}, r\right)$ and $\left(e_{j}, r\right)$. The topology is constructed by adding edges between entity node $e_{i}$ and relation node $\left(e_{i}, r\right)$. Besides, five more networks, including Cornell, Texas, Washington, Wisconsin 


\begin{tabular}{lrrrr}
\hline Methods & Cora & Citeseer & Pubmed & NELL \\
\hline MLP & $55.1 \%$ & $46.5 \%$ & $71.4 \%$ & $22.9 \%$ \\
ManiReg & $59.5 \%$ & $60.1 \%$ & $70.7 \%$ & $21.8 \%$ \\
SemiEmb & $59.0 \%$ & $59.6 \%$ & $71.7 \%$ & $26.7 \%$ \\
LP & $68.0 \%$ & $45.3 \%$ & $63.0 \%$ & $26.5 \%$ \\
DeepWalk & $67.2 \%$ & $43.2 \%$ & $65.3 \%$ & $58.1 \%$ \\
ICA & $75.1 \%$ & $69.1 \%$ & $73.9 \%$ & $23.2 \%$ \\
Planetoid & $75.7 \%$ & $64.7 \%$ & $77.2 \%$ & $61.9 \%$ \\
Chebyshev & $81.2 \%$ & $69.8 \%$ & $74.4 \%$ & - \\
GCN & $81.5 \%$ & $70.3 \%$ & $79.0 \%$ & $66.0 \%$ \\
MoNet & $81.7 \%$ & $69.9 \%$ & $78.8 \%$ & $64.2 \%$ \\
GAT & $83.0 \%$ & $72.5 \%$ & $79.0 \%$ & - \\
\hline DSP-GCN & $\mathbf{8 5 . 0 \%}$ & $\mathbf{7 4 . 2 \%}$ & $\mathbf{8 1 . 2 \%}$ & $\mathbf{6 7 . 3 \%}$ \\
\hline
\end{tabular}

Table 2: Transductive node classification results.

and Wiki, are employed. Four of them, i.e., Texas, Cornell, Washington and Wisconsin, are the sub-networks of the WebKB network. Each of them is the collection of the webpages from an university in U.S.. Similarly, nodes in Wiki network are the webpages from Wikipedia.

\subsection{Methods}

For comparisons, we employ 11 state-of-the-art semisupervised node classification algorithms, including multilayer perceptron (MLP), label propagation (LP) [Zhu et al., 2003], semi-supervised embedding (SemiEmb) [Weston et al., 2012], manifold regularization (ManiReg) [Belkin et al., 2006], graph embedding (DeepWalk) [Perozzi et al., 2014], iterative classification algorithm (ICA) [Lu and Getoor, 2003], graph-based semi-supervised learning framework (Planetoid) [Yang et al., 2016], graph convolution with Chebyshev filters [Defferrard et al., 2016], graph convolutional network (GCN) [Kipf and Welling, 2017], mixture model networks (MoNet) [Monti et al., 2017], and graph attention networks (GAT) [Veličković et al., 2018]. Besides, to give a comprehensive understanding, we also compare DSP-GCN with 5 community detection methods on the attributed networks. Degree-corrected Stochastic Block Model (DCSBM) [Karrer and Newman, 2011] and NetMRF [He et al., 2018] only adopt the network topology, while PCLDC [Yang et al., 2009], SCI [Wang et al., 2016] and NEMBP [He et al., 2017] utilize both the network topology and node attributes. All the results of the baselines are produced by running the codes from the authors with their default settings.

\subsection{Results and Analysis}

In the experiments, DSP-GCN is compared to 11 baseline methods on three citation networks by following the experiment protocols in [Yang et al., 2016], where 20 nodes per class, 500 nodes and 1000 nodes are employed for training, validation and performance evaluation, respectively. On the extracted bipartite graph from NELL, we follow the settings in [Yang et al., 2016] where the label rate is $0.1 \%$. The performance is measured with accuracy (AC). As shown in Table 2, our DSP-GCN outperforms all the baseline methods. The improvement of DSP-GCN compared to GAT, which achieves the best performance among the baseline methods, is moderately significant. Although the performance gain of accuracy is $1.8 \%$ in average, a large proportion of the error rates has been reduced. Specifically, the average error rates of GAT and our proposed DSP-GCN on citation networks are $21.84 \%$ and $19.87 \%$, respectively, Then the error rate reduction, which is achieved by the proposed DSP-GCN, is $(21.84 \%-19.87 \%) / 21.84 \%=9.02 \%$. It clearly demonstrates the effectiveness of our DSP-GCN on jointly exploiting the node content and network topology, which reduces the distortions to the attributes induced by the topology.

To comprehensively evaluate the proposed DSP-GCN, we compare it with five methods, which can also be regarded as node classification techniques. In addition to the three citation networks, five webpage networks, which is often adopted to evaluate the community detection methods, are employed. For the four medium webpage networks, including Cornell, Texas, Washington and Wsicsonsin, we adopt $20 \%$ labelled nodes for training, $10 \%$ labelled nodes for validation, and the other nodes for testing. For the large Wiki network, the percentages of nodes for training and validation are $3 \%$ and $3 \%$, respectively. The results are shown in Table 3 . The performance is measured with accuracy (AC) and normalized mutual information (NMI). As can be observed, our DSP-GCN gives relatively consistent performances and significant improvements on the five webpage networks.

The extensive results have demonstrated the superiority and effectiveness of the proposed DSP-GCN under various circumstances compared to 16 baseline methods. On the other hand, the original GCN gives unsatisfactory results (comparable/worse performances compared to the community detection methods) on the webpage networks. The unstable performances of GCN have revealed that the simple attribute smoothing based on the topology may cause severe degradations to the attributes, thus induce an unsatisfactory prediction result. Besides, the NMI scores of GCN, which are more sensitive to the prediction results of the classes containing very few nodes than $\mathrm{AC}$, are obviously worse than $\mathrm{AC}$ on the webpage networks. This phenomenon indicates that the prediction results of $\mathrm{GCN}$ for the categories possessing very few nodes are unsatisfactory [Zhou et al., 2018], which indicates a severe degradations of the attributes on these rare nodes after the attribute smoothing. Meanwhile, DSP-GCN can obtain satisfactory results on rare node classifications.

\subsection{Case Study}

To further demonstrate the negative effects caused by the topology in classification, a case study on the Citeseer network is carried out. In this experiment, $25 \%$ of the misclassified nodes (74 nodes) in GCN can be correctly classified with respect to the attributes only, where the classification is performed by a single fully-connected layer. This number is reduced to $10 \%$ (27 nodes) in our proposed DSP-GCN. The significant gain of our DSP-GCN verifies that our DSP-GCN can successfully reduce the attribute distortions caused by the topology. 
Proceedings of the Twenty-Eighth International Joint Conference on Artificial Intelligence (IJCAI-19)

\begin{tabular}{|c|c|c|c|c|c|c|c|c|c|c|c|c|c|c|}
\hline \multirow{2}{*}{$\begin{array}{l}\text { Datasets } \\
\text { Criterion }\end{array}$} & \multicolumn{2}{|c|}{ DCSBM } & \multicolumn{2}{|c|}{ NetMRF } & \multicolumn{2}{|c|}{ PCLDC } & \multicolumn{2}{|c|}{ SCI } & \multicolumn{2}{|c|}{ NEMBP } & \multicolumn{2}{|c|}{ GCN } & \multicolumn{2}{|c|}{ DSP-GCN } \\
\hline & $\mathrm{AC}$ & NMI & $\mathrm{AC}$ & NMI & $\mathrm{AC}$ & NMI & $\mathrm{AC}$ & NMI & $\mathrm{AC}$ & NMI & $\mathrm{AC}$ & NMI & $\mathrm{AC}$ & NMI \\
\hline Texas & 48.1 & 16.6 & 30.6 & 5.5 & 38.8 & 10.4 & 49.7 & 12.5 & 53.6 & 35.1 & 57.1 & 5.0 & 72.1 & 52.0 \\
\hline Cornell & 37.9 & 9.7 & 31.8 & 7.3 & 30.3 & 7.2 & 36.9 & 6.8 & 47.2 & 18.7 & 46.3 & 9.1 & 64.1 & 39.0 \\
\hline Washington & 31.8 & 9.9 & 35.0 & 5.8 & 30.0 & 5.7 & 46.1 & 6.8 & 42.9 & 21.1 & 54.9 & 10.5 & 69.9 & 40.2 \\
\hline Wisconsin & 32.8 & 3.1 & 28.6 & 3.2 & 30.2 & 5.0 & 46.4 & 13.3 & 63.4 & 38.0 & 55.6 & 18.8 & 73.1 & 54.4 \\
\hline Wiki & 2.6 & 31.2 & 31.1 & 25.8 & 28.8 & 26.9 & 29.5 & 23.4 & 46.3 & 47.2 & 16.4 & 3.7 & 51.2 & 49.2 \\
\hline CiteSeer & 26.6 & 4.1 & 22.2 & 1.2 & 24.9 & 3.0 & 34.4 & 9.2 & 49.5 & 24.3 & 70.3 & 45.4 & 74.2 & 48.7 \\
\hline Cora & 38.5 & 17.1 & 58.1 & 37.2 & 34.1 & 17.5 & 41.7 & 17.8 & 57.6 & 44.1 & 81.5 & 62.5 & 85.0 & 67.1 \\
\hline PubMed & 53.6 & 12.3 & 55.5 & 16.9 & 63.6 & 26.8 & - & - & 65.7 & 28.3 & 79.0 & 28.0 & 81.2 & 46.2 \\
\hline
\end{tabular}

Table 3: Comparisons with different kinds of community detection methods.

\section{Conclusions}

Topology information is a double-edged sword for GCNN based semi-supervised node classification. It can be exploited to alleviate the problem of attribute sparsity by attribute smoothing over the graph. On the other hand, its employment in the attribute smoothing also degrades the discriminabilities of the attributes. To reduce its distortions while exploit more potentials of the attribute information, we propose Dual SelfPaced Graph Convolutional Network (DSP-GCN) in this paper. It consists of two highly correlated strategies, node-level and edge-level self-paced learning strategies, which gradually add the unlabelled nodes with confidently predicted labels and the simple edges into training, respectively. Extensive experiments on real networks have demonstrated the superiority of the proposed DSP-GCN compared to the baseline methods and its ability to reduces the attribute distortions induced by topology with only one graph convolutional layer.

\section{Acknowledgments}

This work was supported in part by the National Key R\&D Program of China (No.2017YFC0820106), in part by the National Natural Science Foundation of China under Grant 61503281 and Grant 61802391, in part by the Foundation for Innovative Research Groups through the National Natural Science Foundation of China under Grant 61421003, and in part by the Fundamental Research Funds for the Central Universities.

\section{References}

[Belkin et al., 2006] Mikhail Belkin, Partha Niyogi, and Vikas Sindhwani. Manifold regularization: A geometric framework for learning from labeled and unlabeled examples. JMLR, 7:2399-2434, 2006.

[Bengio et al., 2009] Yoshua Bengio, Jérôme Louradour, Ronan Collobert, and Jason Weston. Curriculum learning. In $I C M L$, pages 41-48, 2009.

[Carlson et al., 2010] Andrew Carlson, Justin Betteridge, Bryan Kisiel, Burr Settles, Estevam R. Hruschka Jr., and Tom M. Mitchell. Toward an architecture for never-ending language learning. In $A A A I$, pages 1306-1313, 2010.
[Chapelle et al., 2009] Olivier Chapelle, Bernhard Scholkopf, and Alexander Zien. Semi-supervised learning (chapelle, o. et al., eds.; 2006)[book reviews]. IEEE TNN, 20(3):542-542, 2009.

[Defferrard et al., 2016] Michaël Defferrard, Xavier Bresson, and Pierre Vandergheynst. Convolutional neural networks on graphs with fast localized spectral filtering. In NIPS, pages 3837-3845, 2016.

[Gao and Huang, 2018] Hongchang Gao and Heng Huang. Self-paced network embedding. In $A C M$ SIGKDD, pages 1406-1415, 2018.

[Hamilton et al., 2017] William L. Hamilton, Zhitao Ying, and Jure Leskovec. Inductive representation learning on large graphs. In NIPS, pages 1025-1035, 2017.

[He et al., 2017] Dongxiao He, Zhiyong Feng, Di Jin, Xiaobao Wang, and Weixiong Zhang. Joint identification of network communities and semantics via integrative modeling of network topologies and node contents. In $A A A I$, pages 116-124, 2017.

[He et al., 2018] Dongxiao He, Xinxin You, Zhiyong Feng, Di Jin, Xue Yang, and Weixiong Zhang. A networkspecific markov random field approach to community detection. In $A A A I$, pages 306-313, 2018.

[Karrer and Newman, 2011] Brian Karrer and M. E. J. Newman. Stochastic blockmodels and community structure in networks. PRE, 83:016107, 2011.

[Kipf and Welling, 2017] Thomas N. Kipf and Max Welling. Semi-supervised classification with graph convolutional networks. In ICLR, 2017.

[Kumar et al., 2010] M. Pawan Kumar, Benjamin Packer, and Daphne Koller. Self-paced learning for latent variable models. In NIPS, pages 1189-1197, 2010.

[Li et al., 2018] Qimai Li, Zhichao Han, and Xiao-Ming Wu. Deeper insights into graph convolutional networks for semi-supervised learning. In $A A A I$, pages 3538-3545, 2018.

[Lu and Getoor, 2003] Qing Lu and Lise Getoor. Link-based classification. In ICML, pages 496-503, 2003. 
[Ma et al., 2017] Fan Ma, Deyu Meng, Qi Xie, Zina Li, and Xuanyi Dong. Self-paced co-training. In ICML, pages 2275-2284, 2017.

[Monti et al., 2017] Federico Monti, Davide Boscaini, Jonathan Masci, Emanuele Rodolà, Jan Svoboda, and Michael M. Bronstein. Geometric deep learning on graphs and manifolds using mixture model cnns. In IEEE CVPR, pages 5425-5434, 2017.

[Perozzi et al., 2014] Bryan Perozzi, Rami Al-Rfou, and Steven Skiena. Deepwalk: online learning of social representations. In ACM SIGKDD, pages 701-710, 2014.

[Sen et al., 2008] Prithviraj Sen, Galileo Namata, Mustafa Bilgic, Lise Getoor, Brian Gallagher, and Tina EliassiRad. Collective classification in network data. AI Magazine, 29(3):93-106, 2008.

[Veličković et al., 2018] Petar Veličković, Guillem Cucurull, Arantxa Casanova, Adriana Romero, Pietro Liò, and Yoshua Bengio. Graph attention networks. In ICLR, 2018.

[Wang et al., 2016] Xiao Wang, Di Jin, Xiaochun Cao, Liang Yang, and Weixiong Zhang. Semantic community identification in large attribute networks. In $A A A I$, pages 265-271, 2016.

[Weston et al., 2012] Jason Weston, Frédéric Ratle, Hossein Mobahi, and Ronan Collobert. Deep learning via semisupervised embedding. In Neural Networks: Tricks of the Trade - Second Edition, pages 639-655. 2012.

[Yang et al., 2009] Tianbao Yang, Rong Jin, Yun Chi, and Shenghuo Zhu. Combining link and content for community detection: a discriminative approach. In $A C M$ SIGKDD, pages 927-936, 2009.

[Yang et al., 2016] Zhilin Yang, William W. Cohen, and Ruslan Salakhutdinov. Revisiting semi-supervised learning with graph embeddings. In ICML, pages 40-48, 2016.

[Zhao et al., 2015] Qian Zhao, Deyu Meng, Lu Jiang, Qi Xie, Zongben Xu, and Alexander G. Hauptmann. Selfpaced learning for matrix factorization. In $A A A I$, pages 3196-3202, 2015.

[Zhou et al., 2018] Dawei Zhou, Jingrui He, Hongxia Yang, and Wei Fan. SPARC: self-paced network representation for few-shot rare category characterization. In $A C M$ SIGKDD, pages 2807-2816, 2018.

[Zhu et al., 2003] Xiaojin Zhu, Zoubin Ghahramani, and John D. Lafferty. Semi-supervised learning using gaussian fields and harmonic functions. In ICML, pages 912-919, 2003.

[Zhu, 2006] Xiaojin Zhu. Semi-supervised learning literature survey. Computer Science, University of WisconsinMadison, 2(3):4, 2006. 